\title{
UNFOLDINGS OF HOLOMORPHIC FOLIATIONS
}

\author{
XAVIER GÓMEZ-MONT
}

\begin{abstract}
Absiract
The objective of this paper is to give a criterium for an unfolding of a holomorphic foliation with singularities to be holomorphically trivial.
\end{abstract}

\section{Introduction}

An unfolding is a holomorphic foliation with singularities $\mathcal{F}$ in the complex manifold $M$, with $\pi: M \rightarrow \Delta_{1} \subset C$ a smooth holomorphic map, defining a family of foliations $\mathcal{F}_{t}$ in the family of complex manifolds $M_{t}=\pi^{-1}(t)$, obtained by intersecting the leaves of $\mathcal{F}$ with $M_{t}$ (see definitions 2.1 and 3.1 ), such that the leaves of $\mathcal{F}$ are not contained in the fibres of $\pi$. The leaves of $\mathcal{F}$ have one more dimension than the leaves of $\mathcal{F}_{t}$, and the problem we are addressing in this paper is to give a criterim to determine when the family of foliations $\mathcal{F}_{t}$ is trivial, that is, they are all biholomorphically equivalent. QThe technique that we will use to analyse this problem, will consist in measuring the obstructions to lifting the vector field $\frac{\partial}{\partial t}$ on $\Delta_{1}$ to a holomorphic vector field $Y$ tangent to the foliation $\mathcal{F}$. If $\pi$ is a proper map, or a germ of a map, then the 1-parameter group obtained by integrating $Y$ gives the triviality of the unfolding.

We begin by showing in Proposition 2.2 that the local existence of a lifting of $\frac{\partial}{\partial t}$ is equivalent to the triviality of the extension class of the normal sheaves (i.e. the normal sheaf of the family of foliations $\mathcal{F}_{t}$ is a direct sum of the nomal sheaf of the foliation $\mathcal{F}$ by the structure sheaf). We do not need the integrability of the foliation for this first step. In Theorem 3.3 we use the integrability conditions, and the assumption that the foliation is full, to show that the triviality of the extension class of the conormal sheaves is equivalent to the local triviality of the unfolding. We globalize this criterium in Theorem 4.1.

Having formulated the triviality of the unfolding in terms of a triviality of an extension class, we use a spectral sequence to divide this second problem into two problems: The first is local and concentrated in the singularities of the foliation, and the second is global and measured by a first cohomology group. In section 5 we apply the above procedure to foliations by curves. We show that the local obstructions vanish if the depth of the singularity set is at least 3 , and that the global obstructions are measured by a cohomology group of an invertible sheaf, which may then be analysed by standard vanishing theorems (Cartan-Sere's Theorem B or Kodara-Nalano). In particular we obtain: 
Theorem 5.3. Let $X_{0}: \mathcal{L}_{0} \longrightarrow \Theta_{M_{0}}$ be a foliation by curves with isolated singularities in the compact manifold $M_{0}$. Assume that $M_{0}$ has dimension at least 3 and $H^{1}\left(M_{0}, \mathcal{L}_{0}\right)=0$; then every unfolding of $X_{0}$ is trivial.

We finish by analysing the case of foliations by curves in surfaces:

Theorem 5.5. [G-O] Let $X_{\mathfrak{0}}: \mathcal{L}_{0} \longrightarrow \Theta_{M_{0}}$ be a foliation by curves with isolated singularities in the compact complex 2-dimensional manifold $M_{0}$. Assume that $H^{1}\left(M_{0}, \mathcal{L}_{0}\right)=0$ and that every singular point has multiplicity 1 and non-vanishing trace; then every unfolding of $X_{0}$ is trivial.

The method we present in this paper is a generalization of an idea of Hiashenko [I]. He originally applied it to foliations by curves in the complex projective plane tangent to a line, where after taking affine coordinates of the complement of the line he applied Noether's $A F+B G$ Theorem (see $[G-H] p$. 703) instead of carrying out the cohomological computation.

I would like to thank I. Girbau, M. Nicolau and R. Castelet for providing a stimulating atmosphere during the elaboration of this research at the Centre de Recerca Matemàtica at the Universitat Autònoma de Barcelona, in Catalunya.

\section{Local unfoldings of distributions}

Let

$$
\pi: \mathbb{C} \times \mathbb{C}^{n} \longrightarrow \mathbb{C}, \quad \pi(t, z)=t
$$

with $t \in \mathbb{C}$ and $z=\left(z_{1}, \ldots, z_{n}\right) \in \mathbb{C}^{n}$, be the projection of $\mathbb{C} \times \mathbb{C}^{n}$ to the first factor. $\pi$ induces a ring homomorphism

$$
\pi^{*}: \mathcal{O}_{1} \longrightarrow \mathcal{O}_{n+1}
$$

from the ring of germs of holomorphic functions at 0 in $C$ to the ring of holomorphic functions at $0=(0,0)$ in $\mathbb{C}^{n+1}$. If we denote by $\Omega_{n+1}$ and $\Omega_{1}$ the (free) modules of germs of holomorphic 1 -forms on $\mathbb{C}^{n+1}$ and $C$, respectively, and by $\pi^{*} \Omega_{1}$ the $\mathcal{O}_{n+1}-$ module $\Omega_{1} \otimes \mathcal{O}_{2} \mathcal{O}_{n+1}$, then the pull back of 1 -forms under $\pi$ extends to an exact sequence of $\mathcal{O}_{n+1}$-modules

$$
0 \longrightarrow \pi^{*} \Omega_{1} \longrightarrow \Omega_{n+1} \stackrel{\rho}{\longrightarrow} \Omega_{\pi} \longrightarrow 0
$$

where the right hand term is the (free) $\mathcal{O}_{n+1}$-module of relative differential 1 -forms to $\pi$ and $\rho$ is the projection map.

An element $\vartheta$ of $\Omega_{n+1}$ may be writter as $\vartheta=b(t, z) d t+\sum_{j=1}^{n} a_{j}(t, z) d z_{j}$ and it may be interpreted as describing a possibly singular distribution of codimension one planes in some open neighbourhood of 0 in $\mathrm{C}^{n+1}$. Its projection 
$\rho(\vartheta)$ may be interpreted as a 1-parameter family of distributions defined in neighbourhoods of 0 in $C^{n}$ by $\sum_{j=1}^{n} a_{j}(t, z) d z_{j}$ and it describes the family of distributions obtained by intersecting the distribution defined by $\vartheta$ with the hyperplanes tangent to the $\pi$-fibres.

Definition 2.1. A germ of a family of (singular holomorphic) distributions is an injective $\mathcal{O}_{n+1}$-module map $\bar{\omega}$ defined from an $\mathcal{O}_{n+1}$-module $F$ into $\Omega_{\pi}$, and giving rise to the short exact sequence of $\mathcal{O}_{n+1}$-modules:

$$
0 \longrightarrow F \stackrel{\ddot{m}}{\longrightarrow} \Omega_{\pi} \longrightarrow \Omega_{\pi} / \bar{\omega}(F) \longrightarrow 0
$$

An unfolding of a germ of a family of distributions $\bar{\omega}: F \longrightarrow \Omega_{\pi}$ is a lifting of $\bar{\omega}$ to an $\mathcal{O}_{n+1}$ morphism $\omega: F \longrightarrow \Omega_{n+1}$. We will also call an injective morphism $\omega: F \longrightarrow \Omega_{n+1}$ a germ of a (singtalar holomorphic) distribution and if $\bar{\omega}=\rho \circ \omega$ is an injective $\mathcal{O}_{n+1}$-module map, we will call $\omega$ a germ of an unfolding with $\bar{\omega}$ as underlying family of distributions.

Remark: The recquirement that $\bar{\omega}$ is an injective map means that the image of $\omega$ does not contain elements of the form $b(t, z) d t$, or equivalently, that the planes defined by the unfolding are not all contained in the tangent planes to the $\pi$-fibres.

An unfolding gives rise to an exact commutative diagram of $\mathcal{O}_{n+1}$-modules:

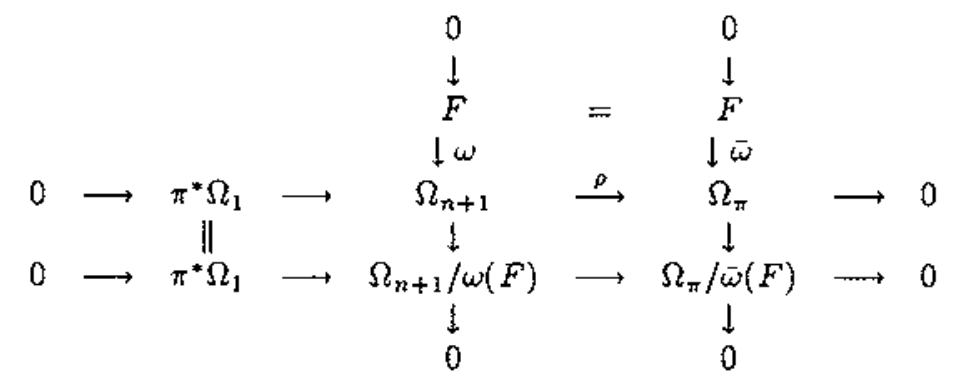

Proposition 2.2. Let $\bar{\omega}: F \longrightarrow \Omega_{\pi}$ be a family of distributions, then:

1) There is a one to one correspondence between the unfoldings of $F$ and the elements in $\operatorname{Hom}\left(F, \pi^{*} \Omega_{1}\right) \cong F^{*}$.

2) The map which associates to each unfolding $\eta \oplus \bar{\omega}$ the extension class of $\Omega_{\pi} / \bar{\omega}(F)$ by $\pi^{*} \Omega_{1}$ in the lower row of $(2.3)$ is the coboundary morphism $\delta$ of a long exact sequence of Ext-groups.

3) If the above extension class $\delta(\eta)$ is 0 , then we may find a germ of a holomorphic vector field tangent to the distribution of the form

$$
\hat{X}=\frac{\partial}{\partial t}-\sum_{j=1}^{n} X_{j}(t, z) \frac{\partial}{\partial z_{j}}
$$


Proof: Since the middle vertical sequence splits, $\Omega_{n+1}=\pi^{*} \Omega_{1} \oplus \Omega_{\pi}$, we may write $\omega=\eta \oplus \bar{\omega}$, where $\eta \in H o m\left(F, \pi^{*} \Omega_{1}\right) \cong F^{*}$. This proves part 1 .

The long exact sequence of Ext-modules associated to the functor $\operatorname{Hom}\left(\cdot, \pi^{*} \Omega_{1}\right)$ applied to the right hand column in (2.3) is:

$$
\begin{aligned}
& 0 \longrightarrow \operatorname{Hom}\left(\Omega_{\pi} / \bar{\omega}(F), \pi^{*} \Omega_{1}\right) \longrightarrow \operatorname{Hom}\left(\Omega_{\pi}, \pi^{*} \Omega_{1}\right) \stackrel{\gamma}{\longrightarrow} \\
& H o m\left(F, \pi^{*} \Omega_{1}\right) \stackrel{\delta}{\longrightarrow} \operatorname{Ext}^{1}\left(\Omega_{\pi} / \bar{\omega}(F), \pi^{*} \Omega_{1}\right) \stackrel{\epsilon}{\longrightarrow} \operatorname{Ext} t^{1}\left(\Omega_{\pi}, \pi^{*} \Omega_{1}\right) \longrightarrow
\end{aligned}
$$

The kernel of $\epsilon$ corresponds to those extensions that induce the trivial extension of $\Omega_{\pi}$ by $\pi^{*} \Omega_{1}$, and these extensions are described as images of $\delta$ by means of elements $\eta \in \operatorname{Hom}\left(F, \pi^{*} \Omega_{1}\right)$. This proves part 2 .

If $\delta(\eta)=0$, then by the exactness of (2.5), we may find an extension $\tilde{\eta}$ : $\Omega_{\pi} \longrightarrow \pi^{*} \Omega_{1}$ of $\eta: F \longrightarrow \pi^{*} \Omega_{1}$; that is, there is a relative vector field

$$
X=\sum_{j=1}^{n} X_{j} \frac{\partial}{\partial z_{j}} \in \Theta_{\pi}=\operatorname{Hom}\left(\Omega_{\pi}, O_{n+1}\right) \cong \operatorname{Hom}\left(\Omega_{\pi}, \pi^{*} \Omega_{1}\right)
$$

such that if for $f \in F$ we have $\bar{\omega}(f)=\sum a_{j}(f) d z_{j}$ then

$$
\begin{gathered}
\omega(f)=\eta(f) d t+\sum a_{j}(f) d z_{j}= \\
\left(\sum a_{j}(f) d z_{j}\right) \circ\left(\sum X_{j} \frac{\partial}{\partial z_{j}}\right) d t+\sum a_{j}(f) d z_{j}
\end{gathered}
$$

where $o$ evaluates a 1 form on a vector field through the natural duality. Hence we have

$$
\omega(f)=\left(\sum a_{j}(f) X_{j}\right) d t+\sum a_{j}(f) d z_{j}
$$

Define now $\tilde{X}$ as in (2.4) using the previously obtained components $X_{j}$, and then

$$
\omega(f) \circ \tilde{X}=\sum a_{j}(f) X_{j}-\sum a_{j}(f) X_{j}=0
$$

Hence $\dot{X}$ is tangent to the distribution defined by $\omega(f)$ for every $f \in F$, so $\dot{X}$ is tangent to the distribution defined by $\omega$. This finishes the proof of the proposition.

To further exploit the vector field (2.4), we need to impose the integrability conditions to the distribution. Although the statement (Theorem 3.3) is local, the proof we present is semi-local; so we will begin the next section with global definitions of foliations and unfoldings of foliations. 


\section{Local unfoldings of foliations}

Let $\pi: M \longrightarrow C$ be a smooth holomorphic map between the complex manifold $M$ of dimension $n+1$ and the complex line $\mathbb{C}$. The fibers of $\pi^{-1}(t)=M_{t}$ of $\pi$ are smooth complex manifolds of dimension n. If $M_{0}$ is compact, we will also assume that $\pi$ is a proper map onto its inage. Overall, we will be interested in a neighbourhood of $M_{0}$, so we consider $\mathbb{C}$ as a pointed space $(\mathbb{C}, 0)$, and we shrink the neighbourhoods around $M_{0}$.

We will denote by

$$
\mathcal{O}_{M}, \Omega_{M}, \Theta_{M}, \Omega_{\pi}, \Theta_{\pi}
$$

the sheaves on $M$ of germs of holomorphic functions, 1-forms, vector fields, relative 1 -forms, relative vector fields, and

$$
\Omega_{M}^{*}=\bigoplus_{p=0}^{n+3} \bigwedge^{p} \Omega_{M}, \quad \Omega_{\pi}^{*}=\bigoplus_{p=0}^{n} \bigwedge^{p} \Omega_{\pi} \text { and } \pi^{*} \Omega_{\varepsilon}=\Omega_{\mathcal{C}} \otimes_{\mathcal{O}_{\mathcal{C}}} \mathcal{O}_{M}
$$

where the first two are the sheaves of exterior algebras of differential and relative differential 1-forms, respectively, with derivatives $d$ and $d_{\pi}$, where $d$ is a $\mathbb{C}$ linear map and $d_{\pi}$ is $\mathcal{O}_{\mathbb{C}}$-linear. Given a subset $A \subset \Omega^{*}$, we will denote by $A \cdot \Omega^{*}$ the sheaf of ideals generated by the elements of $A$.

We obtain an exact sequence of sheaves on $M$, as in (2.1)

$$
0 \longrightarrow \pi^{*} \Omega_{\mathbb{C}} \longrightarrow \Omega_{M} \stackrel{\rho}{\longrightarrow} \Omega_{\pi} \longrightarrow 0
$$

Definition 3.1. A family of distributions (or a distribution) on $M$ is an injective $\mathcal{O}_{M}$-morphism $\bar{\omega}: \mathcal{F} \longrightarrow \Omega_{\pi}$ (respectively, an injective $\mathcal{O}_{M}$-morphism $\left.\omega: \mathcal{F} \longrightarrow \Omega_{M}\right)$ from the coherent sheaf $\mathcal{F}$ on $M$. The singular set Sing $(\bar{\omega})$ (or Sing $(\omega)$ ) of the family of distributions (or of the distribution) is the set of points where $\Omega_{\pi} / \bar{\omega}(\mathcal{F})$ (or $\Omega_{M} / \omega(\mathcal{F})$ ) is not locally free.

A family of foliations (or a foliation, or an unfolding of a family of foliations) is a family of distributions $\bar{\omega}: \mathcal{F} \longrightarrow \Omega_{\pi}$ such that on the non-singular points of $\omega(\mathcal{F})$ we have $d_{\pi}(\omega(\mathcal{F})) \subset \omega(\mathcal{F}) \cdot \Omega_{\pi}^{*}$ (respectively, a distribution $\omega$ : $\mathcal{F} \longrightarrow \Omega_{M}$ such that on the non-singular points of $\omega(\mathcal{F})$ we have $d(\omega(\mathcal{F})) \subset$ $\omega(\mathcal{F}) \cdot \Omega_{M}^{*}$, and for an unfolding we recquire that $\omega$ is a foliation and the morphism of $\mathcal{O}_{M}$-sheaves $\bar{\omega}=\rho \circ \omega: \mathcal{F} \longrightarrow \Omega_{\pi}$ is injective).

We also say in the preceeding definition that the germs of distributions are involutive. We will always consider an unfolding of a family of foliations as being involutive. An unfolding on $M$ gives rise to an exact commutative diagram of $\mathcal{O}_{M}$-modules: 


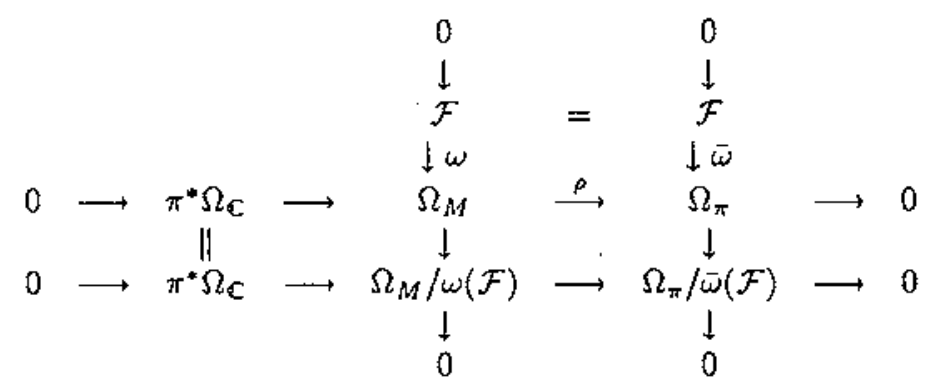

The process of restriction to the fibre $M_{t}$ is carried out by tensoring with $\otimes_{\mathcal{O}_{\mathbb{C}}} C_{t}$, where $\mathcal{C}_{t}=\mathcal{O}_{\mathbb{C}} / m_{t}$ and $m_{t}$ is the sheaf of ideals in $\mathcal{O}_{\mathbb{C}}$ associated to the point $t \in C$. If $\bar{\omega}: \mathcal{F} \longrightarrow \Omega_{\pi}$ is a family of foliations, then its restriction to $M_{t}$ is

$$
\bar{\omega}_{t}: \mathcal{F} \otimes \mathcal{O}_{\mathcal{C}} C_{t} \longrightarrow \Omega_{\pi} \otimes_{\mathcal{O}_{\mathcal{c}}} C_{t} \cong \Omega_{M_{t}}
$$

which may be considered as an $\mathcal{O}_{M_{t}}$-morphism, and after dividing by its kernel (if it is not injective) it defines a foliation on $M_{1}$.

Given $\bar{\omega}_{0}: \mathcal{F}_{0} \longrightarrow \Omega_{M_{0}}$ a foliation on $M_{0}$, its trivial extension to a family of foliations is defined on $\pi: M_{0} \times \mathcal{C} \longrightarrow \mathcal{C}$ by

$$
\bar{\omega}=\bar{\omega}_{0} \otimes_{\mathcal{C}}^{i d}: \mathcal{F}_{0} \otimes_{\mathcal{C}} \mathcal{O}_{\mathbb{C}} \longrightarrow \Omega_{M_{0}} \otimes_{\mathcal{C}} \mathcal{O}_{\mathbb{C}} \cong \Omega_{\pi}
$$

and its trivial extension as an unfolding by further defining $\omega=\eta \oplus \bar{\omega}$ with $\eta=0$.

If $\vec{\omega}: F \longrightarrow \Omega_{\pi}$ (or $\omega: F \longrightarrow \Omega_{n+1}$ ) is a germ of a family of foliations (or a foliation or an unfolding), then it extends to a family of foliations (or a foliation or an unfolding) in a neighbourhood of 0 . This may be realized by extending a set of generators of $F$. By coherence, this extension is unique in a perhaps smaller neighbourhood of 0 .

Definition 3.2. Two unfoldings $\omega_{j}: \mathcal{F}_{j} \longrightarrow \Omega_{M^{j}, j}=1,2$ (or families of foliations $\omega_{j}: \mathcal{F}_{j} \rightarrow \Omega_{\pi_{j}}$ ) are isomorphic if there is a biholomorphisms $\Phi: M^{1} \longrightarrow M^{2}$ over $\mathbb{C}$ (i.e. it induces the identity on C) such that $\omega_{1}\left(\mathcal{F}_{1}\right)=\Phi^{*} \omega_{2}\left(\mathcal{F}_{2}\right)$, where $\Phi^{*}$ is the natural action of $\Phi$ on 1 -forms (respectively $\bar{\omega}_{1}\left(\mathcal{F}_{1}\right)=\Phi^{*} \bar{\omega}_{2}\left(\mathcal{F}_{2}\right)$ ). We say that an unfolding $\omega: \mathcal{F} \longrightarrow \Omega_{M}$ is trivial over 0 (or that a family of foliations $\bar{\omega}$ is trivial over 0 ) if $\omega$ (or $\bar{\omega}$, respectively) is isomorphic to the trivial extension of the restriction of $\omega$ to $M_{0}$ in some neighbourhood of the fibers over $0 \in \mathbb{C}$. A germ of an unfolding at $0 \in C \times C^{n}$ is trivial if there is a neighbourhood of 0 where the unfolding is trivial.

The singular set Sing $(\bar{\omega})$ of a family of foliations $\bar{\omega}: \mathcal{F} \longrightarrow \Omega_{\pi}$ is defined as the set of points where $\Omega_{\pi} / \bar{\omega}(\mathcal{F})$ is not locally free. The singular set Sing $(\omega)$ 
of an unfolding or of a foliations $\omega: \mathcal{F} \longrightarrow \Omega_{M}$ is defined as the set of points where $\Omega_{M} / \omega(\mathcal{F})$ is not locally free. By the Theorem of Frobenius (see $[\mathrm{CH}]$ ), we may find at a non-singular point of $\bar{\omega}$ (or of $\omega$ ) cocrdinates $\left(t, w_{1}, \ldots, w_{n}\right)$ such that $\bar{\omega}(\mathcal{F}) \subset \Omega_{\pi}$ (respectively $\omega(\mathcal{F}) \subset \Omega_{M}$ ) is generated by $d w_{1}, \ldots, d w_{q}$. With these coordinate charts, that are called foliated coordinate charts, we may define on $M-\operatorname{Sing}(\bar{\omega})$ (or $M-\operatorname{Sing}(\omega)$ ) a geometric foliation $F o l(\omega)$ (or $F$ ol $(\bar{\omega})$ ) that gives a decomposition of $M-S i n g(\bar{\omega})$ into a disjoint union of connected complex submanifolds, which in general are only inmersed and called the leaves of the foliation, that are locally defined by $w_{j}=K_{j}, j=1, \ldots, q$.

We may associate to a family of foliations $\bar{\omega}: \mathcal{F} \longrightarrow \Omega_{\pi}$ a foliation on the total space $M$ which has the same leaves as $F o l(\bar{\omega})$. This is done by taking the inverse inage of $\bar{\omega}$ in (3.1) to obtain an exact commutative diagram:

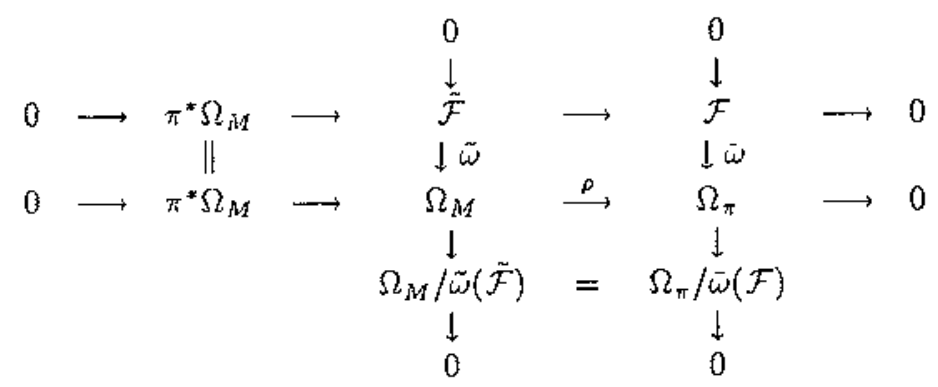

An $\mathcal{O}_{n+1}$-submodule $G$ of $\Omega_{n+1}$ (or of $\Omega_{\pi}$ ) is full if $G$ is equal to its double dual $G^{* *}$, where the duality is the one between 1 -forms and vector fields, (or between relative 1 -forms and relative vector fields, respectively). We will say that a germ of a family of foliations $\vec{\omega}: F \longrightarrow \Omega_{\pi}$ (or a germ of a foliation $\omega: F \longrightarrow \Omega_{n+1}$ ) is full if $\bar{\omega}(F) \subset \Omega_{\pi}$ (or $\omega(F) \subset \Omega_{n+1}$ ) is a full submodule. Similarly an $\mathcal{O}_{M}$-submodule of $\Omega_{M}, \ldots$, etc. is full if the stalk over every point of $M$ is full. The fullness condition is useful, since a full foliation is completely determined by the foliation at the non-singular points.

Theorem 3.3. Let $w: F \longrightarrow \Omega_{n+1}$ be a germ of a full unfolding of a family of foliations $\bar{\omega}: F \longrightarrow \Omega_{\pi} . \omega$ is a trivial unfolding if and only if there is an $\mathcal{O}_{n+1} \cdot$ module isomorphism

$$
\Omega_{n+1} / \omega(F) \cong \pi^{*} \Omega_{1} \oplus \Omega_{\pi} / \bar{\omega}(F)
$$

Proof: If $\omega$ is a trivial unfolding, then we may find a neighbourhood and a biholomorphism over $\pi$ such that $\omega$ has the form $\omega=0 \oplus \bar{\omega}_{0}$, where $\bar{\omega}_{0}$ is the induced foliation in $\{0\} \times \mathrm{C}^{n}$, as in (3.3). In these coordinates, the extension class in Proposition 2.2 is $\delta(0)=0$; hence we have (3.5). 
Conversely, assume that (3.5) holds. This condition means that the extension class in Proposition 2.2 is 0 , and so by Proposition 2.2 we may find a germ of a holomorphic vector field $\tilde{X}$ as in (2.4) tangent to the distribution. Extend everything to a neighbourhood of 0 .

By integrating $\dot{X}$ with initial conditions on $\{0\} \times C^{n}$ we obtain a biholomorphism over $\mathbb{C}$. Using this biholomorphism as a change of coordinates, we may assume that $\dot{X}$ is $\frac{\partial}{\partial t}$. In the following argument we will make use of the integrability conditions. We will first argue at the nonsingular points of the foliation, and then use the fullness hypothesis to extend the conclusion to the singular points.

Restrict to an open set $U=\Delta_{1} \times \Delta_{n}$ of $\mathrm{C} \times \mathrm{C}^{n}$ which is the product of small disks around the origin. Let $V$ be a connected open subset in $U-\operatorname{Sing}(\omega)$ such that for every $z \in \Delta_{n}$ we have that $V \cap \pi_{2}^{-1} \pi_{2}(z)$ is connected, where $\pi_{2}: \Delta_{1} \times \Delta_{n} \longrightarrow \Delta_{n}$ is the projection to the second factor. We claim that $\pi_{2}^{-1} \pi_{2}(V)$ is contained in $U-\operatorname{Sing}(\omega)$ and that the unfolding there is the pullback of a non-singular foliation in $\pi_{2}(V)$

To see this, observe that $F o l(\omega)$ in $V$ is non-singular and tangent to $\frac{\partial}{\partial t}$, so if we intersect $V$ with a set of the form $\{t\} \times \Delta_{n}$, it will induce a nonsingular foliation there, of the same codimension. Project it to $\pi_{2}(V)$. These foliations coincide for distinct values of $t$, since the foliation is tangent to $\frac{\partial}{\partial t}$ and $V \cap \pi_{3}^{-1}(z)$ is connected. By pulling back this foliation, we obtain two foliations in $\pi_{2}^{-1} \pi_{2}(V)$, one of them non-singular, the other one full, and they coincide on an open non-empty subset. By the principle of analytic continuation, they. coincide at the non-singular points of $F o l(\omega)$; but since $\omega$ is full, actually they coincide. This proves the claim.

If we denote by $\dot{\omega}$ the trivial extension as an unfolding to the restriction of $\omega$ to $\{0\} \times \Delta_{n}$, as in $(3.3)$, the preceeding argument shows that $F o l(\omega)$ and $F_{0} l(\bar{\omega})$ have the same singular set, and that they agree at the non-singular points. Since $\omega$ is full by hypothesis, this implies that we have the sheaf inclusion

$$
\tilde{\omega}\left(\left(\mathcal{F} \otimes_{\mathcal{O}_{v}} \mathcal{C}_{0}\right) \otimes_{\mathrm{C}} \mathcal{O}_{\mathrm{C}}\right) \subset \omega(\mathcal{F})
$$

We must still show that they coincide. Let $\vartheta$ be an element in the right hand side of (3.6). Expand $\theta$ as a power series in $\mathrm{t}$

$$
\vartheta(t, z)=\sum_{j=1}^{n} \vartheta_{j}(z) t^{j}
$$

where the $\vartheta_{j}(z)$ are holomorphic 1-forms on $\Delta_{n}$. We claim that each $\vartheta_{j}$ belongs to the left hand side of (3.6). To see this, observe that $\vartheta_{0}$ is the restriction of $\vartheta$ to $0 \times \Delta_{n}$. Hence by definition it lies on the left hand side of (3.6). Now $t^{-1}\left(\vartheta-\vartheta_{0}\right)$ 
belongs to the right hand side of (3.6) on the complement of $\{0\} \times \Delta_{n}$, so by fullness also on all of $U$. Repeating the argument for this new 1-form, we obtain that $\vartheta_{1}$ also belongs to the left hand side of (3.6). By induction then, all $\vartheta_{j}$ belong to the left hand side of (3.6). By properties of closures under convergence in compact sets of sections of a coherent sheaf, see [G-R], we then have that the sum (3.7) also lies in the left hand side of (3.6). This proves equality, and hence the theorem.

Proposition 3.4. Let $\omega: \mathcal{F} \longrightarrow \Omega_{M}$ be an unfolding of a family of foliations $\bar{\omega}: \mathcal{F} \longrightarrow \Omega_{\pi}$. Then

1) $\operatorname{Sing}(\omega) \subset \operatorname{Sin} g(\bar{\omega})$

2) Sing $(\bar{\omega})-\operatorname{Sing}(\omega)$ is the set of points $p$ of $M-\operatorname{Sing}(\omega)$ such that the leaf of Fol( $\omega)$ through $p$ is tangent to $M_{\pi(p)}$ at $p$.

Proof: 1) We prove that $M-\operatorname{Sing}(\bar{\omega}) \subset M-\operatorname{Sing}(\omega)$. At a non-singular point of $\bar{\omega}$ we have that $\left(\Omega_{\pi} / \bar{\omega}(\mathcal{F})\right)_{p}$ is locally free, but since all extensions of locally free modules by locally free modules are trivial (i.e. $E x t^{1}\left(\left(\Omega_{\pi} / \bar{\omega}(\mathcal{F})\right)_{p}\right.$, $\left.\left(\pi^{*} \Omega_{C}\right)_{p}\right)=0$ ), we also have by $(2.3)$ that $\left(\Omega_{M} / \omega(\mathcal{F})\right)_{p}$ is free. Hence $p \in$ $M-\operatorname{Sing}(\omega)$.

2) Let $p \in M-\operatorname{Sing}(\omega)$. Then $\mathcal{F}_{p}$ is locally free at $\mathrm{p}$, and so is $\tilde{\mathcal{F}}_{p}$ in (3.4). The map $\bar{\omega}: \tilde{\mathcal{F}} \longrightarrow \Omega_{M}$ in (3.4) has the same cokernel sheaf as $\bar{\omega}: \mathcal{F} \longrightarrow$ $\Omega_{\pi}$, so we are looking for a criterium under which $\left(\Omega_{M} / \tilde{\omega}(\tilde{\mathcal{F}})\right)_{p}$ is free. Let $d t, \vartheta_{1}, \ldots, \vartheta_{q}$ be a basis for $\dot{\omega}(\tilde{\mathcal{F}})_{p}$. The point $\mathrm{p}$ is non-singular for $F o l(\tilde{\omega})$ if and only if $d t(p), \vartheta_{1}(p), \ldots, \vartheta_{q}(p)$ are linearly independent as elements in the cotangent space to $M$ at $p$. Since $p \notin \operatorname{Sing}(\omega)$, we have that $\vartheta_{1}(p), \ldots, \vartheta_{q}(p)$ are linearly independent; so $p$ is non-singular for $F o l(\tilde{\omega})$ if and only if the leaf of $F o l(\omega)$ through $p$ is not tangent to $M_{\pi(p)}$ at $p$. This proves the proposition.

\section{Global unfoldings of foliations}

Let $\pi: M \longrightarrow \Delta_{1}$ be a proper smooth holomorphic map onto the the unit disk $\Delta_{1}$ in $\mathcal{C}$, and let $\omega: \mathcal{F} \longrightarrow \Omega_{M}$ be an unfolding of a family of holomorphic foliations $\bar{\omega}: \mathcal{F} \longrightarrow \Omega_{\pi}$. The lower row in (3.2) shows that $\Omega_{M} / \omega(\mathcal{F})$ is an extension of $\Omega_{\pi} / \bar{\omega}(\mathcal{F})$ by $\pi^{*} \Omega_{\Delta_{1}}$. Recall (see [G-H] p.705 or [H-2] p.237) that these extension classes are classified by elements in the global $\operatorname{Ext} t^{1}\left(M ; \Omega_{\pi} / \bar{\omega}(\mathcal{F}), \pi^{*} \Omega_{\Delta_{1}}\right)$ group.

Theorem 4.1. Let $\pi: M \longrightarrow \Delta_{1}$ be a proper and smooth morphism onto the unit disk, $\Delta_{1}$ in $\mathbb{C}$, and let $\omega: \mathcal{F} \longrightarrow \Omega_{M}$ be a full unfolding of a family of foliations $\bar{\omega}: \mathcal{F} \longrightarrow \Omega_{\pi}$. $\omega$ is a trivial unfolding over $0 \in \Delta_{1}$ if and only if after shrinking the disk $\Delta_{1}$ there is an $\mathcal{O}_{M}$-sheaf isomorphism

$$
\Omega_{M} / \omega(\mathcal{F}) \cong \pi^{*} \Omega_{\Delta_{1}} \oplus \Omega_{\pi} / \bar{\omega}(\mathcal{F})
$$


Proof: If $\omega$ is a trivial unfolding over 0 , then we may find a neighbourhood of $M_{0}$ and a biholomorphism over $\pi$ such that $\omega$ has the form $\omega=0 \oplus \bar{\omega}_{0}$, where $\bar{\omega}_{0}$ is the induced foliation in $M_{0}$, as in (3.3). After applying this isomorphism, we clearly have (4.1).

Conversely, assume that (4.1) holds. There is a long exact sequence of global Ext groups associated to the functor $\operatorname{Hom}\left(M ;-, \pi^{*} \Omega_{\Delta_{1}}\right)$ applied to the right hand column in (3.2):

$$
\begin{array}{cccc}
0 \longrightarrow & \operatorname{Hom}\left(M ; \Omega_{\pi} / \bar{\omega}(\mathcal{F}), \pi^{*} \Omega_{\Delta_{1}}\right) & \rightarrow & \operatorname{Hom}\left(M ; \Omega_{\pi}, \pi^{*} \Omega_{\Delta_{1}}\right) \\
\stackrel{\gamma}{\longrightarrow} & H o m\left(M ; \mathcal{F}, \pi^{*} \Omega_{\Delta_{1}}\right) & \stackrel{6}{\longrightarrow} & \mathrm{E}_{x} t^{1}\left(M ; \Omega_{\pi} / \bar{\omega}(\mathcal{F}), \pi^{*} \Omega_{\Delta_{1}}\right) \\
\stackrel{\epsilon}{\longrightarrow} & \operatorname{Ext}^{1}\left(M ; \Omega_{\pi}, \pi^{*} \Omega_{\Delta_{1}}\right) & \longrightarrow &
\end{array}
$$

There is a canonical element $\xi \in \mathrm{E} x t^{1}\left(M ; \Omega_{\pi}, \pi^{*} \Omega_{\Delta_{1}}\right)$ which represents $\Omega_{M}$ as the extension in the middle row of (3.2). Recalling that $\mathrm{E} x t^{1}\left(M ; \Omega_{\pi}, \pi^{*} \Omega_{\Delta_{1}}\right)$ is isomorphic to $H^{1}\left(M, \Theta_{\pi}\right)$, we see that this element is the Kodaira-Spencer class of the family of varieties $\left\{M_{t}\right\}$ (see $[\mathrm{K}-\mathrm{S}]$ ). Using the isomorphism between this extension and $\Omega_{M}$, we see that $\epsilon^{-1}(\xi)$ in (4.2) represent all the unfoldings of $\bar{\omega}$ as a family of distributions, as in diagram (3.2).

The hypothesis (4.1) is then equivalent, after shrinking $\Delta_{I}$ to a smaller disk, to having the unfolding $\omega$ represented in $\operatorname{Ext}_{x}{ }^{1}\left(M ; \Omega_{\pi} / \bar{\omega}(\mathcal{F}), \pi^{*} \Omega_{\Delta_{1}}\right)$ by the 0 element. Since $\epsilon(0)=0$, we deduce that the family $\pi$ is the trivial family: $M=\Delta_{1} \times M_{0}$. In this case, by the conmutativity of (4.2), we may represent all unfoldings by means of elements of $\operatorname{Hom}\left(M ; \mathcal{F}, \pi^{*} \Omega_{\Delta_{1}}\right)$. Since by hypothesis the unfolding $\omega$ is inducing the trivial extension, again by the exactness of (4.2) we may find an element

$$
X \in H^{0}\left(M, \Theta_{\pi}\right) \cong H o m\left(M ; \Omega_{\pi}, \pi^{*} \Omega_{\Delta_{l}}\right)
$$

The holomorphic vector field $\vec{X}=\frac{\partial}{\partial t}-X$ is, as in the proof of Proposition 2.2 and Theorem 3.3, tangent to the unfolding $\omega$ and its integral 1-parameter group sends $\pi$-fibres to $\pi$-fibres. We may then use this vector field, as in the proof of Proposition 2.2 and Theorem 3.3, to show that the unfolding is trivial. This proves the Theorem.

In order to compute if an unfolding induces a trivial extension (4.1), we may use one of the spectral sequences associated to the global Ext construction, which gives an exact sequence:

$$
\begin{aligned}
0 \longrightarrow H^{1}(M, \mathcal{T}) & \longrightarrow \operatorname{Ext}^{1}\left(M ; \Omega_{\pi} / \bar{\omega}(\mathcal{F}), \pi^{*} \Omega_{\Delta_{1}}\right) \\
& \stackrel{\rho}{\longrightarrow} H^{0}\left(M ; \mathcal{E} x t^{1}\left(\Omega_{\pi} / \bar{\omega}(\mathcal{F}), \pi^{*} \Omega_{\Delta_{1}}\right)\right)
\end{aligned}
$$


where $T$ is the sheaf of germs of relative holomorphic vector fields tangent to the family of foliations $\bar{w}$, whose stalk over $p \in M$ is

$$
\tau_{p}=\left\{Y \in \Theta_{\pi, p} / \bar{\omega}(f) \circ X=0, \forall f \in \mathcal{F}_{p}\right\}
$$

It is the annihilator subsheaf of $\mathcal{F}$ under the natural pairing of $\Omega_{\pi}$ and $\Theta_{\pi}$. The sheaf $\mathcal{E} x t^{1}\left(\Omega_{\pi} / \bar{\omega}(\mathcal{F}), \pi^{*} \Omega_{\Delta_{1}}\right)$ has as stalks the local $\mathcal{E} z t^{1}$ discussed in section 2 , and it has support on $\operatorname{Sing}(\bar{\omega})$. The map $\rho$ associates to an extension $e$ the local invariants $\rho(e)_{p}$ that measure the non-triviality of the extensions at the points $p$ of $M$. The kernel of $\rho$ consists of those extensions that are locally a direct sum at every point of $\mathrm{M}$, and are parametrized by $H^{1}(M, T)$.

\section{Unfoldings of holomorphic foliations by curves}

In this section we obtain a criterium for a foliation by curves to have only trivial unfoldings.

Definition 5.1. A family of foliations by curves in $\pi: M \longrightarrow \Delta_{1} \subset \subset$ may be defined by a holomorphic map $X: \mathcal{L} \longrightarrow \Theta_{\pi}$ from an invertible sheaf $\mathcal{L}$ on $M$ to the relative tangent bundle $\Theta_{\pi}$, such that $X$ does not vanish identically along a $\pi$-fibre. The coherent sheaf $\mathcal{F}$ defined as the kernel of the sheaf map obtained by dualising $X$ :

$$
0 \longrightarrow \mathcal{F} \stackrel{\stackrel{u}{\longrightarrow}}{\longrightarrow} \Omega_{\pi} \stackrel{x^{*}}{\longrightarrow} \mathcal{L}^{*}
$$

defines the family of foliations associated to $X$, in the sense of definition 3.1. $B y$ an unfolding of the family of foliations by curves $X$, we understand an unfolding of $\bar{\omega}$. An unfolding of a foliation by curves $X_{0}: \mathcal{L}_{0} \longrightarrow \Theta_{M_{0}}$ in the manifold $M_{0}$ is an unfolding of any family of foliations by curves $X: \mathcal{L} \longrightarrow \Theta_{\pi}$ on $M, \pi: M \longrightarrow \Delta_{1} \subset C$ a smooth holomorphic map such that $\pi^{-1}(0)=M_{0}$ and $X_{\mid M_{0}}=X_{0}$. If $M_{0}$ is compact, we assume that the map $\pi$ is proper.

The singular set $S$ of the family of foliations by curves defined by $(5.1)$ is the subspace of $M$ defined locally by the ideal $\left(X_{1}, \ldots, X_{n}\right) \subset \mathcal{O}_{U}$, where

$$
X=\sum_{j=1}^{n} X_{j}(t, z) \frac{\partial}{\partial z_{j}}
$$

is an expression of $X$ in local coordinates of $U \subset M$, after having trivialized $\mathcal{L}$, $\mathcal{L}=\mathcal{O}_{U}$. We may form two short exact sequences from $(5.1)$ :

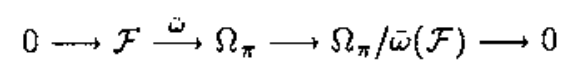

and

$$
0 \longrightarrow \Omega_{\pi} / \bar{\omega}(\mathcal{F}) \longrightarrow \mathcal{L}^{*} \longrightarrow \operatorname{Cok} X^{*} \longrightarrow 0
$$


where $\Omega_{\pi} / \bar{\omega}(\mathcal{F})=\operatorname{Im}_{m} X^{*}$ is locally isomorphic to the ideal generated by $\left(X_{1}, \ldots, X_{n}\right) \subset \mathcal{O}_{U}=\mathcal{L}_{\mid U}$, where we are using the expression (5.2). $\operatorname{Cok} X^{*}$ is a sheaf supported on $S$.

Recall that a complex analytic subspace $V \subset M$ in the complex manifold $M$ has depth at least $r$ at $p$ if we may find germs $f_{1}, \ldots, f_{r}$ of holomorphic functions at $p$ belonging to the ideal defining $V$ such that they form a regular sequence, namely for $j=2, \ldots, r$ we have that $f_{j}$ is not a divisor of zero in $\mathcal{O}_{p} /\left(f_{1}, \ldots, f_{j-1}\right)$, (see $[\mathrm{G}-\mathrm{H}]$, p. 688 , or $[\mathrm{H}]$, p. 36$)$.

Theorem 5.2. Let $X: \mathcal{L} \longrightarrow \Theta_{\pi}$ define a family of holomorphic foliations by curves $\bar{\omega}: \mathcal{F} \longrightarrow \Omega_{\pi}$ in $\pi: M \longrightarrow \Delta_{1} \subset \mathcal{C}$. If the singular set $S=\{X=0\}$ has depth at least 3 in $M$, then:

$$
\mathcal{E}_{x t^{1}}\left(\Omega_{\pi} / \bar{\omega}(\mathcal{F}), \pi^{*} \Omega_{\Delta_{1}}\right)=0
$$

and

$$
\mathrm{E}_{x} t^{1}\left(M ; \Omega_{\pi} / \bar{\omega}(\mathcal{F}), \pi^{*} \Omega_{\Delta_{1}}\right)=H^{1}(M, \mathcal{L})
$$

If we further assume that $\pi$ is a proper map and $H^{1}\left(M_{0}, \mathcal{L}_{0}\right)=0$, where $\mathcal{L}_{0}$ is the restriction of $\mathcal{L}$ to $M_{0}=\pi^{-1}(0)$, then every unfolding of $X$ is trivial.

Proof: Let $U$ be an open subset of $M$ where the foliation is defined by the vector field $X$ as in (5.2). Denote by $I$ the sheaf of ideals on $U$ generated by $\left(X_{1}, \ldots, X_{n}\right)$. The hypothesis that $S$ has depth at least 3 is equivalent (see $[H]$, p.39) that for $j=0,1,2$ we have on $U$

$$
\mathcal{E}_{x t_{O_{U}}^{j}}\left(\mathcal{O}_{U} / \mathcal{I}, \mathcal{O}_{U}\right)=0
$$

Consider the long exact sequence of $\mathcal{E} x t$-sheaves associated to the functor Kom $\left(*, O_{U}\right)$ applied to

$$
0 \longrightarrow \mathcal{I} \longrightarrow \mathcal{O}_{U} \longrightarrow \mathcal{O}_{U} / \mathcal{I} \longrightarrow 0
$$

From it, and the above observation, we deduce that $\mathcal{E} x t^{1}\left(\mathcal{I}, \mathcal{O}_{U}\right)=0$. This proves (5.5). We deduce now (5.6) from (5.5) and (4.3).

The last assertion follows from (5.6), since by upper-semicontinuity of cohomology for flat families of sheaves, (see [H-2], p. 288 and 250) and the hypothesis $H^{1}\left(M_{0}, \mathcal{L}_{0}\right)=0$ implies $H^{1}(M, \mathcal{L})=0$.

Theorem 5.3. Let $X_{0}: \mathcal{L}_{0} \longrightarrow \Theta_{M_{0}}$ be a foliation by curves with isolated singularities in the compact manifold $M_{0}$. Assume that $M_{0}$ has dimension at least 3 and $H^{1}\left(M_{0}, \mathcal{L}_{0}\right)=0$; then every unfolding of $X_{0}$ is trivial.

Proof: Let $X: \mathcal{L} \longrightarrow \Theta_{r}$ be a family of foliations by curves that extend $X_{0}$, and let $X_{t}$ be its restriction to $M_{t}$. The singular set $S$ of $X$ has dimension 1 , since its intersection with the fibres $M_{t}$ is a finite number of points $([\mathbf{G}-\mathbf{H}], \mathrm{p}$. $662)$. Hence $S$ is locally a complete intersection, and so Theorem 5.2 applies.

As a particular case we obtain: 
Corollary 5.4. Let $X_{0}$ be a homogeneous polynomial vector field inducing a foliation by curves in the projective space $C P^{n}, n \geq 3$, with isolated singularities; then any unfolding of $X_{0}$ is trivial.

Proof: By a Theorem of Serre ([H-2], p. 225) for any invertible sheaf $\mathcal{L}_{0}$ on $C P^{n}, n \geq 2$, we have $H^{1}\left(C P^{n}, \mathcal{L}_{0}\right)=0$. Hence Corollary 5.3 applies.

We say that a singularity $p$ of a foliation by curves $X_{0}: \mathcal{L}_{0} \rightarrow \Theta_{M_{0}}$ in $M_{0}$ has non-vanishing trace if the trace of the linear part at $p$ of a vector field $Y_{0}$ defining the foliation in a neighbourhood of $p$ is non-zero; i.e., if $Y_{0}=$ $\sum_{j=1}^{n} Y_{j}(z) \frac{\partial}{\partial z}$, then $\sum_{j=1}^{n} \frac{\partial Y_{j}}{\partial z_{j}}\left(p_{0}\right) \neq 0$. We also say that the singularity $p$ above has multiplicity $l$ if the ideal generated by $Y_{1}, \ldots, Y_{n}$ in the local ring $\mathcal{O}_{M_{0, \gamma}}$ of germs of holomorphic functions on $M_{0}$ at $p$ is the maximal ideal.

Theorem 5.5. Let $X_{0}: \mathcal{L}_{0} \longrightarrow \Theta_{M_{0}}$ be a foliation by curves with isolated singularities in the compact complex 2-dimensional manifold $M_{0}$, such that every singular point has multiplicity 1 , non-vanishing trace, and we have $H^{1}\left(M_{0}, \mathcal{L}_{0}\right)=0$; then every unfolding of $X_{0}$ is trivial.

We will first prove the following:

Lemma 5.6. Let $X_{0}$ be a germ of a holomorphic vector feld in $\left(\mathbb{C}^{2}, 0\right)$, having 0 as a critical point of multiplicity 1 and having non-vanishing trace. Then, any germ of an unfolding of $X_{0}$ as a foliation is trivial.

Proof: A germ of a family of foliations by curves may be described by a germ of a family of vector fields $X_{t}=B\left(z_{1}, z_{2}, t\right) \frac{\partial}{\partial z_{2}}-A\left(z_{1}, z_{2}, t\right) \frac{\partial}{\partial z_{2}}$. Since by hypothesis $X_{0}$ has multiplicity 1 , we may after a change of coordinates assume that 0 is the only singular point of $X_{t}$ and it has multiplicity 1 . This may also be stated by saying that the ideal generated by $A$ and $B$ in $\mathcal{O}_{\mathbb{C}^{3}, 0}$ is $\left(z_{1}, z_{2}\right)$.

A germ of an unfolding of the family of foliations $X_{t}$ may be described by a germ of a holomorphic 1-form $\omega=A\left(z_{1}, z_{2}, t\right) d z_{1}+B\left(z_{1}, z_{2}, t\right) d z_{2}+$ $C\left(z_{1}, z_{2}, t\right) d t$ which satisfies the integrability condition $\omega \wedge d \omega=0$. Writing out the integrability condition, we obtain:

$\omega \wedge d \omega=\left[A\left(-\frac{\partial B}{\partial t}+\frac{\partial C}{\partial z_{2}}\right)-B\left(-\frac{\partial A}{\partial t}+\frac{\partial C}{\partial z_{1}}\right)+C\left(-\frac{\partial A}{\partial z_{2}}+\frac{\partial B}{\partial z_{1}}\right)\right] d z_{1} \wedge d z_{2} \wedge d t=0$

Restricting now to the singular set $S$ of the family of foliations $X_{t}$, defined by $A=B=0$, we obtain on $S$ the equality $C\left(-\frac{\partial A}{\partial z_{2}}+\frac{\partial B}{\partial z_{1}}\right)$. Observe that the factor within the parenthesis is the trace of $X_{t}$, and since we are assuming that it is non-zero, we obtain that $C=0$ restricted to $S$. In terms of ideals, this means that $C$ is a section of the ideal defining $S$ (since $S$ is reduced), so that we may find germs of holomorphic functions $P$ and $Q$ in $\left(C^{3}, 0\right)$ such that $C=P A+Q B$. Now, define a germ of a holomorphic vector field in $\left(C^{3}, 0\right)$ by 
$Y=-P \frac{\partial}{\partial z_{1}}-Q \frac{\partial}{\partial z_{2}}+\frac{\partial}{\partial t}$. We then have that $\omega(Y)=0$, which means that $Y$ is a vector field tangent to the unfolding. Arguing now as we did in Theorem 3.3, we deduce that the germ of the unfolding is trivial. This proves the Lemma.

Proof of the Theorem: Let $\omega$ be an unfolding of the foliation by curves $X_{0}$. By Theorem 4.1, this unfolding is trivial if and only if the associated extension class $e$ in $\operatorname{Ext}^{1}\left(M ; \Omega_{\pi} / \bar{\omega}(\mathcal{F}), \pi^{*} \Omega_{\Delta_{1}}\right)$ is 0 . We use the sequence (4.3) to show that it is 0 .

First, $\rho(e) \in H^{0}\left(M ; \mathcal{E} x t^{2}\left(\Omega_{\pi} / \bar{\omega}(\mathcal{F}), \pi^{*} \Omega_{\Delta_{1}}\right)\right)$ is 0 , since by Lemma 5.6 the unfolding is locally trivial as a germ at every singular point, and this section measures exactly this local obstructions. But then by the exactness of (4.3) we have that $e$ is in the image of $H^{1}(M, T)$. By the upper semicontinuity of cohomology groups in flat families (see $[\mathrm{H}-2]$, p. 288) we have $H^{1}\left(M_{t}, \mathcal{L}_{t}\right)=0$, and then using the degenerate spectral sequence associated to the higher direct image functors of $\pi$ (see $[\mathrm{H}-2]$, p.250), we have that $H^{1}(M, \mathcal{T})=0$. This then shows that $e=0$, and so the unfolding is trivial. This proves the Theorem.

Corollary 5.7. Let $X_{0}$ be a homogeneous polynomial vector field inducing a foliation by curves in the projective plane $C P^{2}$, with only singularities of multiplicity 1 and non-vanishing trace; then any unfolding of $X_{0}$ is trivial.

Proof: By a Theorem of Serre $\left([\mathrm{H}-2]\right.$, p. 225) for any invertible sheaf $\mathcal{L}_{0}$ on $C P^{2}$, we have $H^{1}\left(C P^{2}, \mathcal{L}_{0}\right)=0$. Hence Theorem 5.5 applies.

\section{References}

[Ch] C. Chevalley, C., "Theory of Lie Groups," Princeton U.P., 1946.

[G-O] X. Gomez-MonT, L. ORTiz-Bobadilla, "Sistemas Dinámicos Holomorfos en Superficies," Aportaciones Matemáticas, Serie Investigación 3, 1989.

[G-H] Ph. Griffiths, Ph., J. HARRIS, "Principles of Algebraic Geometry," J.Wiley, 1978.

[G-R] R. GUNming; H. RossI, "Analytic Functions of Several Complex Variables," Prentice-Hall, 1965.

[H] R. HARTShorne, "Local Cohomology," Springer LNM 41, 1967.

[H-2] R. HARTShoRne, "Algebraic Geometry," Springer Graduate Texts, 1974.

[I] J.ILIASHENKO, Topology of phase portraits of analytic differential equations on a complex projective plane, Transl. engl. Selecta Math. Sov. 5, No. 2, (1986), 84-136 (Russian, 1978). 
[K-S] K. KODAIRA, D. SPENCER, On deformations of complex analytic structures, I-II, Ann. of Math. 67 (1958), 328-466.

\author{
CIMAT, A.P. 402 \\ Guanajuato 36000 \\ MÉXICO
}

Rebut el 23 d'Agost de 1989 